\title{
Prospective evaluation of the treatment of solitary rectal ulcer syndrome with biofeedback
}

\author{
C J Vaizey, A J Roy, M A Kamm
}

\begin{abstract}
Background-Solitary rectal ulcer syndrome (SRUS) is often resistant to medical and surgical treatment.

Aim-To determine whether biofeedback retraining is a useful treatment for this condition.

Patients-Thirteen consecutive patients with SRUS (three men, median age 34 years, median duration of symptoms three years) underwent treatment. Previous surgical treatment had failed in five.

Methods-Patients were evaluated prospectively. Anorectal physiological studies were performed in 11 patients before treatment. A standardised questionnaire was used before and after treatment, and all but two patients were examined after treatment.

Results-Median follow up was nine months (range 3-22 months). After treatment four patients were asymptomatic, and four felt improved. Symptom improvement or elimination occurred in: need to strain ( $7 / 13$ patients), digitation (7/11), laxative use (5/9). Time in the toilet (median $30 v 10$ minutes, before $v$ after treatment) and number of visits to toilet (6 $v$ 3/day) were also improved. Three patients were able to maintain employment before treatment compared with eight after treatment. The solitary ulcer did not heal completely in any of the nine patients examined after treatment, but improved in four. Previous surgery, the macroscopic appearance of the ulcer, the presence of pelvic floor paradox, and other physiological parameters did not predict outcome.
\end{abstract}

Conclusion-Biofeedback retraining is a useful treatment for this condition. Long term studies are now required.

(Gut 1997; 41: 817-820)

Keywords: solitary rectal ulcer syndrome; biofeedback therapy

St Mark's Hospital, Northwick Park,

Watford Road, Harrow, Middlesex HA1 3UJ, UK

C J Vaizey

A J Roy

M A Kamm

Correspondence to: Dr Kamm.

Accepted for publication 16 July 1997

The solitary rectal ulcer syndrome (SRUS) is characterised by a combination of symptoms, clinical findings and histological abnormality. Rectal bleeding, disordered defecation and anal pain are associated with a benign rectal lesion with typical histological findings. The macroscopic appearance ranges from hyperaemia to ulceration or even a polypoid lesion, and the lesions are not necessarily solitary. The histological features consist of mucosal thickening with oedema of the lamina propria, fibrosis, and extension of smooth muscle fibres upwards between the crypts. Full thickness rectal histology reveals architectural derangement of the muscularis propria in some patients.

The condition is uncommon and difficult to treat. $^{2}$ Conservative treatments including laxatives, fibre supplementation and attempted reduction of straining are of no proven benefit and there are no topical agents known to improve this condition. Only anteroposterior rectopexy has been reported to improve symptoms in a significant number of patients. ${ }^{3}$ Posterior rectopexy or anterior excision of the rectum have not been associated with encouraging results and in some cases have worsened symptoms. Even rectal excision with coloanal anastomosis is associated with recurrence. Patients rarely require a colostomy.

The cause of this condition is unknown. However, physiological ${ }^{4}$ and histological studies $^{1}$ suggest a spectrum of disease, raising the possibility that this syndrome may result from more than one cause. In clinical practice some patients seem to have a behavioural disorder with excessive straining, whereas in others there is no history of straining. The encouraging results from the use of behavioural therapies for defecation disorders led us to explore whether some patients with SRUS might benefit from biofeedback retraining. There is only one report of the use of biofeedback as the sole treatment for SRUS in adults. ${ }^{5}$ In that study patients were only included if they demonstrated paradoxical puborectalis contraction on testing, and the follow up was less than six months in all cases. The seven patients reported less straining, easier defecation and cessation of bleeding and the passage of mucous after treatment. In another study two children were successfully treated for solitary rectal ulcer using biofeedback training. ${ }^{6}$

Biofeedback has also been used as an adjunct to surgery in this condition. ${ }^{7}$ In one study 17 patients were treated with a combined approach of surgery and biofeedback before or immediately after the surgery. A comparison group of 14 patients treated conservatively or with surgery alone had a higher recurrence rate.

In this prospective study we report the use of biofeedback retraining for SRUS in a consecutive unselected group of adults without restrictive criteria for inclusion.

\section{Patients and Methods}

Thirteen patients (three men, median age 34 years, age range $21-54$ years) were treated. The 
Questions asked when interviewing patients before and after biofeedback therapy

- Number of bowel actions per day

- Number of attempts at passing a stool per day

- Time spent in the toilet trying to pass a stool

- Passage of blood or mucous per rectum

- Need to strain at stool

- Need to digitate per rectum or vagina

- Presence of a feeling of incomplete emptying

- Presence of abdominal bloating

- Presence of pain

- Presence and nature of faecal incontinence

- Details of laxatives taken

- Other medications taken

- Ability to work

median duration of symptoms was three years (range $1-16$ years).

Anorectal physiological studies were undertaken in 11 of the 13 patients before biofeedback treatment, to determine whether any physiological parameter might be a predictor of outcome. The absence of paradoxical contraction did not exclude the patients from biofeedback retraining. Symptoms were also recorded before treatment using the structured questionnaire shown in the box.

Eight patients had biofeedback as their initial treatment and in five patients previous surgery had failed. Of these five patients, one had had an abdominal rectopexy and one had had two abdominal rectopexies; one patient had had an abdominal rectopexy and an anterior resection, one patient had had a Delorme's procedure, and another had had a haemorrhoidectomy. None of the 13 patients had any external rectal prolapse.

Macroscopically, six patients had ulceration, three had polypoid changes and four had hyperaemia. All had characteristic histological changes.

After treatment, 11 patients were reinterviewed and examined. Two patients were unable to re-attend and were interviewed by telephone. All interviews and examinations were undertaken by a doctor who was not the primary caring physician and not the biofeedback therapist.

\section{ANORECTAL PHYSIOLOGY TESTS}

Anorectal physiology studies were performed according to previously published techniques. Anal manometry was performed using a waterfilled microballoon station pull through technique $^{8}$ to measure the maximum resting and voluntary contraction or squeeze pressure. Pudendal nerve terminal motor latency measurements were made bilaterally using a disposable electrode (13L40, Dantec, Skolunde, Denmark) and a nerve stimulating apparatus (Neuromatic 2000M, Dantec). ${ }^{9}$ Rectal thresh- old, urge and maximum tolerated volumes to balloon distension were tested with air inflation of a standard balloon. ${ }^{10}$ Anal and rectal mucosal electrosensitivity thresholds were measured using a bipolar ring electrode (21L11, Dantec) placed $1 \mathrm{~cm}$ above the anal verge, and $6 \mathrm{~cm}$ above the upper limit of the anal canal high pressure zone, respectively. ${ }^{11}$ The presence of paradoxical contraction of the pelvic floor muscles was assessed using adhesive electrodes (Dantec surface electrodes) which were placed over the external sphincter muscles. Muscle activity was recorded during attempted expulsion of a $50 \mathrm{ml}$ water filled balloon.

\section{BIOFEEDBACK THERAPY}

Each patient saw a biofeedback therapist as an outpatient every one to two weeks, usually for four or five sessions. They lay on a couch on their right side facing the therapist and the EMG display unit. A balloon was inserted into the rectum and inflated with $50 \mathrm{ml}$ air so that the patient had the sensation of a full rectum and thus the need to defecate. Two adherent surface electrodes were placed adjacent to the anal opening to assess external anal sphincter function. The patient watched the trace of muscle activity and could see the pattern of electrical activity at rest and during voluntary contraction. The patient was then asked to observe the trace while attempting to expel the balloon. If there was an obvious increase, rather than the normal decrease, in activity, the patient was encouraged to strain without increasing sphincter activity so that its appearance continued to resemble the resting trace.

Patients were also taught how to strain effectively by using a propulsive force through bracing with their abdominal muscles.

They were advised on normal defecatory behaviour and bowel habits. This included restricting the number of visits to the toilet for patients who made frequent defecatory attempts during the day, or increasing the number of visits to the toilet for those patients with infrequent defecation. The amount of time spent, and posture, in the toilet were also specified.

At each biofeedback session the therapist tried to achieve a good rapport with the patient to facilitate good understanding and collaboration. This included gaining an appreciation about the patients personal life and psychological factors which may have been relevant.

An attempt was made to wean patients off laxatives, enemas and suppositories. Sometimes this was achieved early in the course of therapy, and sometimes progressively over a longer period. When the course of biofeedback therapy was complete patients were encouraged to continue practising the techniques they had learnt.

\section{Results}

The median follow up period after the completion of the first course of biofeedback therapy was nine months (range 3-22 months).

The patients symptoms before and after biofeedback therapy are shown in table 1 . When 
TABLE 1 Results of biofeedback therapy for solitary rectal ulcer syndrome in 13 patients

\begin{tabular}{lll}
\hline Variable & Before & After \\
\hline Need to strain & 13 & 6 (decreased in 3 of these) \\
Anal digitation & 11 & 4 (less frequently in 3 of these) \\
Pain & 11 & 8 \\
Sensation of incomplete evacuation & 11 & 8 \\
Visits to toilet/24 hours & Median 6 & Median 3 \\
Time in toilet per visit & Median 30 minutes & Median 10 minutes \\
Passage of blood & $12 / 12$ (1 patient blind) & 5 (less frequently in 2 of these) \\
Passage of mucus & $12 / 12$ (1 patient blind) & 5 (less frequently in 3 of these) \\
Dependence on laxatives & 9 & 4 \\
\hline
\end{tabular}

asked about symptoms after treatment, four patients were asymptomatic, three were much improved, and one was only slightly improved. Of the five patients who failed treatment, two have requested further biofeedback therapy, and two who were thought to have a major psychological component to their symptoms were referred for psychological therapy. The remaining patient had developed symptoms following involvement in the Gulf war and was also thought to require psychological assistance, but declined this.

Prior to treatment three patients were able to maintain employment or study without difficulty, and this increased to eight after treatment.

The macroscopic abnormality did not resolve completely in any of the 11 examined patients, but did improve notably in four. Of these four patients, three had polypoid changes and one had ulceration. Histological studies were not repeated.

PREDICTORS OF OUTCOME

Previous surgical therapy did not preclude benefit from biofeedback therapy. Three of the five patients in whom surgical therapy had failed were improved or asymptomatic after treatment.

Physiological studies were unremarkable in all patients. All patients had a normal resting and squeeze anal pressure, pudendal nerve terminal motor latencies, and anal sensitivity. All patients except one had a normal rectal sensitivity to electrical stimulation. Therefore, no physiological parameter was useful in predicting the response to treatment.

The presence of paradoxical puborectalis contraction on physiological testing before treatment did not predict the response to treatment. Three of five with paradox improved with treatment, as did four out of five patients without. One of the patients who had physiological studies was not tested for paradoxical contraction before treatment.

\section{Discussion}

This study has shown that a behavioural approach seems to have therapeutic benefit for a proportion of patients with this condition. Patients often felt subjectively better, many symptoms associated with bowel function were improved, and some patients were able to work again.

The SRUS may relate to chronic straining in some patients. It would therefore seem sensible to direct treatment towards retraining toiletting behaviour. Biofeedback therapy involves more than just retraining pelvic floor co-ordination. It also teaches patients the necessary posture and use of abdominal muscles during defecation, and imposes a discipline about number of visits to the toilet, time spent in the toilet, digitation, and laxative use. Lastly, it provides them with psychological support. The term "biofeedback" in this context therefore relates to a complex combination of behavioural conditioning, attention to the defecatory process and possibly other psychological effects.

It could be argued that biofeedback is a placebo therapy. However, this condition seems to have a low placebo response rate. In many of the patients treated in this series surgical therapy had failed, and drug therapy had failed in all. An objective measure of benefit was the number of patients able to resume normal employment or studying.

The ulcer did not heal completely in any of our patients. This may relate to inadequate length of follow up. However, it also highlights the disparity between symptoms and the objective mucosal abnormality.

The results of biofeedback should be compared with the results of other treatments. We have recently reviewed the long term results of surgical treatment for this condition at our institution. ${ }^{12}$ Sixty six patients underwent 72 procedures over a 10 years, including rectopexy, Delorme's procedure, and resection. At interview of the patients who had had a rectopexy, $18 \%$ had complete resolution of symptoms, $36 \%$ had notable improvement, $9 \%$ had only an initial improvement, and $36 \%$ had no improvement or felt worse after the operation. No patient had a complete resolution of symptoms after a Delorme's procedure but $56 \%$ were greatly improved, $11 \%$ had only initial improvement and 33\% were unchanged or worse. Coloanal or anterior resection resulted in only a $20 \%$ improvement rate. Overall, $52 \%$ of patients who did not have a stoma were improved. There are few other surgical reports in the literature. In the series by Keighley and Shouler ${ }^{13}$ there were 14 patients with SRUS who underwent abdominal rectopexy. There was a $50 \%$ healing rate of the ulcer. Eight patients had no associated prolapse and of these patients only two had ulcer healing. Tjandra et $a l^{14}$ reported on 31 procedures in 27 patients. There was an overall symptomatic improvement after $65 \%$ of the procedures. Fourteen procedures were performed on patients without prolapse. Only one third of patients had symptomatic improvement after rectopexy, 50\% after resection and $83 \%$ after a "local procedure".

It has been suggested previously that polypoid lesions respond to treatment better than the non-polypoid type. ${ }^{14}$ Some of the patients in our series with a polypoid lesion did fare well with biofeedback therapy, but the number of patients with different types of SRUS was too small to determine whether this was a prognostic factor.

Other non-invasive treatments have also been suggested recently, although they fail to tackle possible underlying aetiology. Their long term benefit is therefore uncertain. They 
include the use of human fibrin sealant on the ulcer $^{15}$ or the excision of the ulcer using NdYAG lasers. ${ }^{16}$

Biofeedback therapy is non-invasive and free of side effects. Surgical treatment is not without associated morbidity, in particular constipation following rectopexy. ${ }^{17}$ We therefore recommend biofeedback therapy as a first line treatment for this condition. In those who do come to operation it may be useful in decreasing postoperative recurrence. ${ }^{6}$ Over the period of the biofeedback therapy course the therapist is also in an optimal situation to assess the patient's psychological status, which may help in selecting which failed patients are suitable for surgical treatment.

1 Kang YS, Kamm MA, Engel AF, Talbot IC. Pathology of the rectal wall in solitary rectal ulcer syndrome and complete rectal prolapse. Gut 1996; 38: 587-90.

2 Lam TCF, Lubowski DZ, King DW. Solitary rectal ulcer syndrome. Baillieres Clin Gastroenterol 1992; 6 : 129-43.

3 Nicholls RJ, Simson JN. Anteroposterior rectopexy in the treatment of solitary rectal ulcer syndrome without overt rectal prolapse. Br F Surg 1986; 73: 222-4.

4 Kang YS, Kamm MA, Nicholls RJ. Solitary rectal ulcer and complete rectal prolapse: one condition or two? Int $\mathcal{f} \mathrm{Colo}-$ rectal Dis 1995; 10: 87-90.

5 Ho YH, Ho JM, Parry BR, et al. Solitary rectal ulcer syndrome: The clinical entity and anorectal physiological findings in Singapore. Aust NZ ₹ Surg 1995; 65: 93-7.
6 Binnie NR, Papachrysostomou M, Clare N, et al. Solitary rectal ulcer: The place of biofeedback and surgery in the treatment of the syndrome. World $\mathcal{F}$ Surg 1992: 16: 836-40.

7 Eigenmann PA, Le Coultre C, Cox J, et al. Solitary rectal ulcer: an unusual cause of rectal bleeding in children. Eur $\mathcal{F}$ Pediatr 1992; 151: 658-60.

8 Rogers J, Laurberg S, Misiewicz J, et al Anorectal physiology validated: a repeatability study of the motor and sensory tests of anorectal function. Br F Surg 1989; 76: 607-9.

9 Kiff ES, Swash M. Normal proximal and delayed distal conduction in the pudendal nerves of patients with idiopathic (neurogenic) faecal incontinence. $\mathcal{F}$ Neurol Neurosurg Psychiatry 1984; 47: 820-3.

10 Farthing MJ, Lennard-Jones JE. Sensibility of the rectum to distension and the anorectal distension reflex in ulcerative colitis. Gut 1978; 19: 64-9.

11 Kamm MA, Lennard-Jones JE. Rectal mucosal electrosensory tests-evidence for a rectal sensory neuropathy in idiosory tests-evidence for a rectal sensory neuropathy in idio-
pathic constipation. Dis Colon Rectum 1990; 33: 419-23.

12 Sitzler PJ, Kamm MA, Nicholls RJ. Surgery for solitary recal ulcer syndrome. Abstracts from the Tripartite meeting. 1996 Jul 8-10; London. Int $\mathcal{F}$ Colorectal Dis 1996; 11: 136.

13 Keighley MR, Shouler P. Clinical and manometric features of the solitary rectal ulcer syndrome. Dis Colon Rectum 1984; 27: 507-12

14 Tjandra JJ, Fazio VW, Church J M, et al. Clinical conundrum of solitary rectal ulcer. Dis Colon Rectum 1992; 35: 227-34.

15 Ederle A, Bulighin G, Orlandi PG, Pilati S. Endoscopic application of human fibrin sealant in the treatment of soliapplication of human fibrin sealant in the treatment of solitary rectal $736-7$.

16 Rau B, Harikrishnan K, Krishna S. Laser therapy of solitary rectal ulcers: a new concept. Ann Acad Med Singapore 1994; 23: $27-8$.

17 Madden MV, Kamm MA, Nicholls RJ, et al. Abdominal rectopexy for complete prolapse: prospective study evaluating changes in symptoms and anorectal function. Dis Colon Rectum 1992; 35: 48-55. 